\title{
Evidence for Luttinger liquid behavior in crossed metallic single-wall nanotubes
}

\author{
B. Gao, ${ }^{1}$ A. Komnik, ${ }^{2}$ R. Egger,${ }^{3}$ D.C. Glattli, ${ }^{1,4}$ and A. Bachtold ${ }^{1}$ \\ ${ }^{1}$ Laboratoire de Physique de la Matière Condensée de l'Ecole Normale Supérieure, 75231 Paris 05, France \\ 2 Physikalisches Institut, Albert-Ludwigs-Universität, 79104 Freiburg, Germany \\ 3 Institut für Theoretische Physik, Heinrich-Heine-Universität, 40225 Düsseldorf, Germany \\ 4 SPEC, CEA Saclay, 91191 Gif-sur-Yvette, France
}

(Dated: September 11, 2018)

\begin{abstract}
Experimental and theoretical results for transport through crossed metallic single-wall nanotubes are presented. We observe a zero-bias anomaly in one tube which is suppressed by a current flowing through the other nanotube. The phenomenon is shown to be consistent with the picture of strongly correlated electrons within the Luttinger liquid model. The most relevant coupling between the nanotubes is the electrostatic interaction generated via crossing-induced backscattering processes. Explicit solution of a simplified model is able to describe qualitatively the observed experimental data with only one adjustable parameter.
\end{abstract}

PACS numbers: 73.63.Fg, 73.50.Fq, 73.23.-b, 73.40.Gk

Single-wall carbon nanotubes (SWNTs) continue to receive a lot of attention in connection to electronic transport in interacting one-dimensional (1D) quantum wires. Metallic SWNTs represent a nearly perfect 1D system, with $\mu$ m-long mean free paths $1,2,2,3]$ and only two spindegenerate transport channels, where it has been theoretically predicted that electrons form a Luttinger liquid (LL) rather than a conventional Fermi liquid phase [4, 5]. Experimental evidence for LL behavior in an individual SWNT has been reported in tunneling 6 , 7, , 8] and resonant tunneling measurements $[9]$, revealing a pronounced suppression in the tunneling density of states [zero-bias anomaly (ZBA)]. Although the observed power-law ZBA can be consistently explained by the LL theory, it is difficult to rule out alternative explanations based on, e.g., environmental dynamical Coulomb blockade. Furthermore, a very similar ZBA has been experimentally observed in multi-wall nanotubes [10,11, 12] although such systems are known to be disordered multi-channel wires [13, 14]. It is therefore of importance to clearly identify Luttinger liquid signatures beyond the ZBA for tunneling into an individual SWNT [6, 7, 8]. Following the proposal of Refs. 15, 16], in this paper we report experimental evidence in support of the LL picture from electrical transport through two crossed metallic SWNTs.

Albeit crossed nanotubes have been investigated by other groups before [17, 18, 19], so far no transport measurements for crossed metallic SWNTs have been reported below room temperature. In our experiments, the conductance is measured first in one tube while the second is left floating. The conductance decreases as the temperature or the bias is reduced, in a way very similar to that of tunneling experiments in SWNTs. Interestingly, the ZBA disappears as the current is increased through the second tube. Below we discuss the relationship between these results and LL predictions. The electrostatic coupling between the tubes is expected to pin the sliding low-energy excitations (plasmons). This mechanism is enhanced by the backscattering generated by the mechanical deformation of the tubes at the cross-
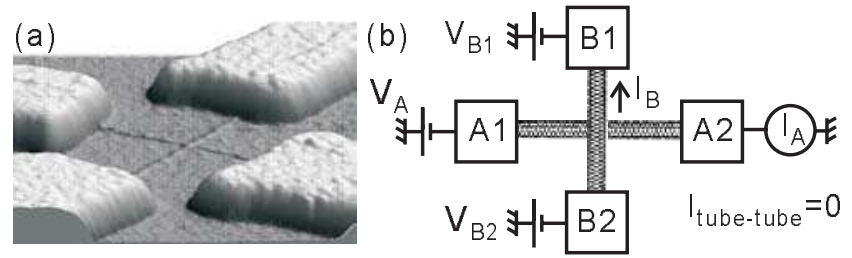

FIG. 1: (a) AFM image of a crossed SWNT junction. The electrode height is $45 \mathrm{~nm}$. (b) Scheme of the device together with the measurement setup. The AFM image can not discriminate which tube lies on top of the other.

ing. When current is imposed to flow in one tube, the pinning tends to be suppressed, enhancing the plasmon sliding and therefore the current in the second tube. Explicit calculations based on LL theory are able to reproduce the measurements rather well, while several alternative explanations are shown to be unlikely below.

The SWNT material is synthesized by laser ablation 20]. SWNTs are dispersed from a suspension in dichloroethane onto an oxidized $\mathrm{Si}$ wafer. $\mathrm{AFM}$ is then used to locate crossed SWNTs with an apparent height of $\approx 1 \mathrm{~nm}$, presumably corresponding to individual SWNTs. Next, Cr/Au electrodes are attached using electron-beam lithography. An example of a device is shown in Fig. 1(a). The separation $L$ between the crossing point and the electrodes is chosen to be $\simeq 300 \mathrm{~nm}$. For shorter $L$, undesired finite-size effects may come into play, while for much longer $L$, the probability is enhanced to find disorder centers along the SWNTs that complicate the analysis. Devices were then studied above $20 \mathrm{~K}$, where the thermal length $\hbar v_{F} / k T$, with Fermi velocity $v_{F}$, remains short compared to $L$. More than 60 samples have been realized, but we have never been lucky enough to achieve a device with two crossed metallic SWNTs and, at the same time, to keep all contact resistances low, so that Coulomb blockade is negligible. Four times an almost ideal device has been obtained, where only one of the four contact resistances was large. Measurements 

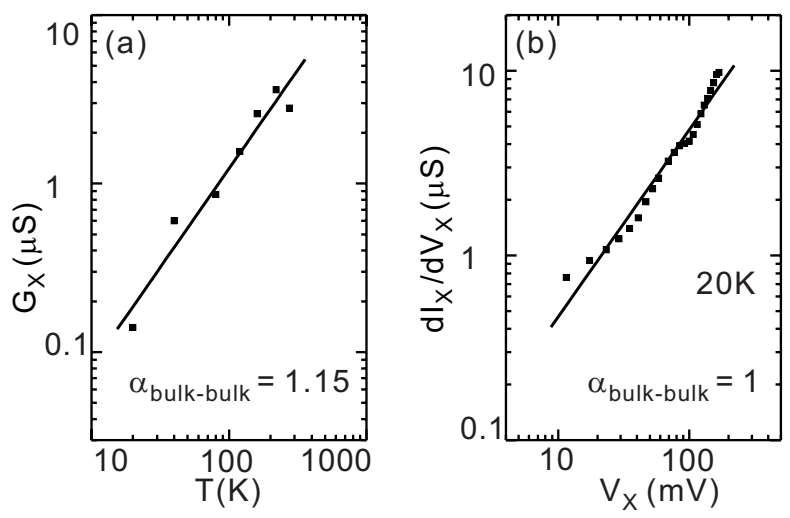

FIG. 2: Tunneling measurement on the tube-tube junction in a four-probe configuration. (a) Linear conductance $G_{X}\left(V_{X}=\right.$ $0, T)$. (b) Differential conductance $d I_{X} / d V_{X}\left(V_{X}, T=20 K\right)$.

have been carried out on these four devices, which all gave similar results. A representative set of measurements on one device is presented next.

In this device, at $T=220 \mathrm{~K}$, the two-point resistances at zero bias of the two SWNTs [henceforth called $A$ and $B]$ are $R_{A}=19 \mathrm{k} \Omega$ and $R_{B}=524 \mathrm{k} \Omega$, while the fourpoint resistance of the tube-tube junction is $R_{X}=277$ $\mathrm{k} \Omega$. Other two-point measurements with electrode $B 1$ contacted, see Fig. 1(b) for the electrode identification, give also large resistance, suggesting that the large $R_{B}$ comes from a poor interface between tube $B$ and electrode $B 1$. Note that the two-point measurements are achieved with the other electrodes left floating. When temperature is decreased, this large contact resistance induces Coulomb blockade (CB) oscillations in tube $B$ with zero current for different regions in the backgate voltage $V_{g}$. In the following, $V_{g}$ is fixed at a broad CB peak.

The device is further characterized by measuring the LL interaction parameter $g$ [4, 5] from the tunneling density of states. Fig. 2 shows the differential tube-tube tunneling conductance $G_{X}\left(V_{X}, T\right)=d I_{X} / d V_{X}$ measured in a four-point configuration. Electrons tunnel from the middle of one SWNT to the middle of the second SWNT (bulk-bulk tunneling). The double-logarithmic plots of $G_{X}\left(V_{X}, T\right)$ in Fig. 2 are in the studied ranges described by a power-law scaling with slope $\alpha_{\text {bulk-bulk }} \simeq 1$.1. Using $\alpha_{\text {bulk-bulk }}=\left(g^{-1}+g-2\right) / 4$ [4, [5], this gives $g \simeq 0.16$. This value is slightly lower than the generally reported values $g \simeq 0.2$ for tunneling into a SWNT from a metal electrode [ 6, 7, 8], reflecting slightly stronger Coulomb interactions among the electrons. This is presumably due to different geometries in Refs. $[6,7,8]$ and in our device, in particular concerning the size and location of the connecting electrodes screening part of the interaction.

Fig. 31a) shows the differential conductance $d I_{A} / d V_{A}$ measured on tube $A$ as a function of $V_{A}$ for different temperatures and with tube $B$ left floating. A clear ZBA is observed, which becomes larger as temperature is decreased. Such a ZBA has been observed many times in
SWNTs [6, 7, 8], and implies that a barrier lies along the tube or at the interface with the electrodes. Fig. [3(b) shows $d I_{A} / d V_{A}\left(V_{A}\right)$ when a current $I_{B}$ is imposed to flow through the second tube. Interestingly, the ZBA is progressively suppressed when $I_{B}$ is increased. We note that the ZBA suppression depends only on the intensity of $I_{B}$ and not on its sign. For these measurements, the sample was biased such that no current flows from tube A to tube $\mathrm{B}$ through the crossing point. In order to achieve this, first a three-point measurement is carried out on tube $A$ under bias $V_{A}$ to determine the potential $V_{A}^{X}$ at the crossing. The voltage drops between the crossing and each electrode are recorded as a function of $V_{A}$ and are found to be half of the bias applied on the tube. In a second step, the three-point measurement is carried out on tube $B$ to record the potential $V_{B}^{X}$ at the crossing as a function of $V_{B}$. This time, the voltage drops are very different on both sides of the tube reflecting the large contact resistance at the $B 1$ electrode. Finally, $I_{A}$ is measured as a function of $V_{A}$ for different $V_{B}$ where voltages $V_{B 1}$ and $V_{B 2}$ applied on electrodes $B 1$ and $B 2$ are continually adjusted so that $V_{A}^{X}=V_{B}^{X}$, see also Fig. 1(b). Since most of $V_{B}$ drops at the bad contact $B 1$, we give instead of $V_{B}$ the current $I_{B}$ in Fig. 3 (b) legend, which is measured while tube $A$ is left floating. The differential conductance is obtained using numerical differentiation.

We review now some possible explanations for the $I_{B^{-}}$ dependence of the ZBA. Let us first consider the effect of Joule heating. Note that heating effects are generally disregarded in tunneling experiments into individual tubes 6, 7, 8, 10, 11, 12]. However, the poor $B 1$ contact releases significant heat in tube $B$. Part of it flows through tube $A$, which may then change the temperature sensitive $G_{A}$. Unfortunately, the temperature rise $\Delta T$ is difficult to estimate, already because little is known about the thermal conductances of individual SWNTs and tubetube junctions. Nevertheless, a qualitative statement can be made. Since $G_{A}(20 \mathrm{~K}, 0.6 \mu \mathrm{A}) \simeq G_{A}(40 \mathrm{~K}, 0 \mathrm{~A})$ and $G_{A}(40 \mathrm{~K}, 0.6 \mu \mathrm{A}) \simeq G_{A}(80 \mathrm{~K}, 0 \mathrm{~A})$, the same heat input $360 \mathrm{nW}$ would give rise to temperature increases $20 \rightarrow 40 \mathrm{~K}$ and $40 \rightarrow 80 \mathrm{~K}$. This would imply that the thermal conductance decreases with $T$, which is very unlikely in this $T$ range [21, 22, 23]. Hence thermal effects alone cannot explain our observations. Another explanation might be related to the capacitive coupling between the tubes. Metal tubes can have an energy dependent conductance, which thus varies with $V_{g}$ as in interference experiments 2, 3]. Here the conductance $G_{A}$ is indeed observed to fluctuate with $V_{g}$. One could thus argue that tube $B$ just acts as a gate. However, the fluctuations with $V_{g}$, which are lower than $2.1 \mu \mathrm{S}$ at $20 \mathrm{~K}$ and above, cannot account for the large modulation of $G_{A}\left(I_{B}\right)$. We conclude that another explanation is needed to account for our experimental results.

Next we compare the data to Luttinger liquid predictions for two crossed SWNTs with identical LL parameter $g$ 15, 16]. Since the experiment is carried out at zero tube-tube current, single-electron tunneling at 

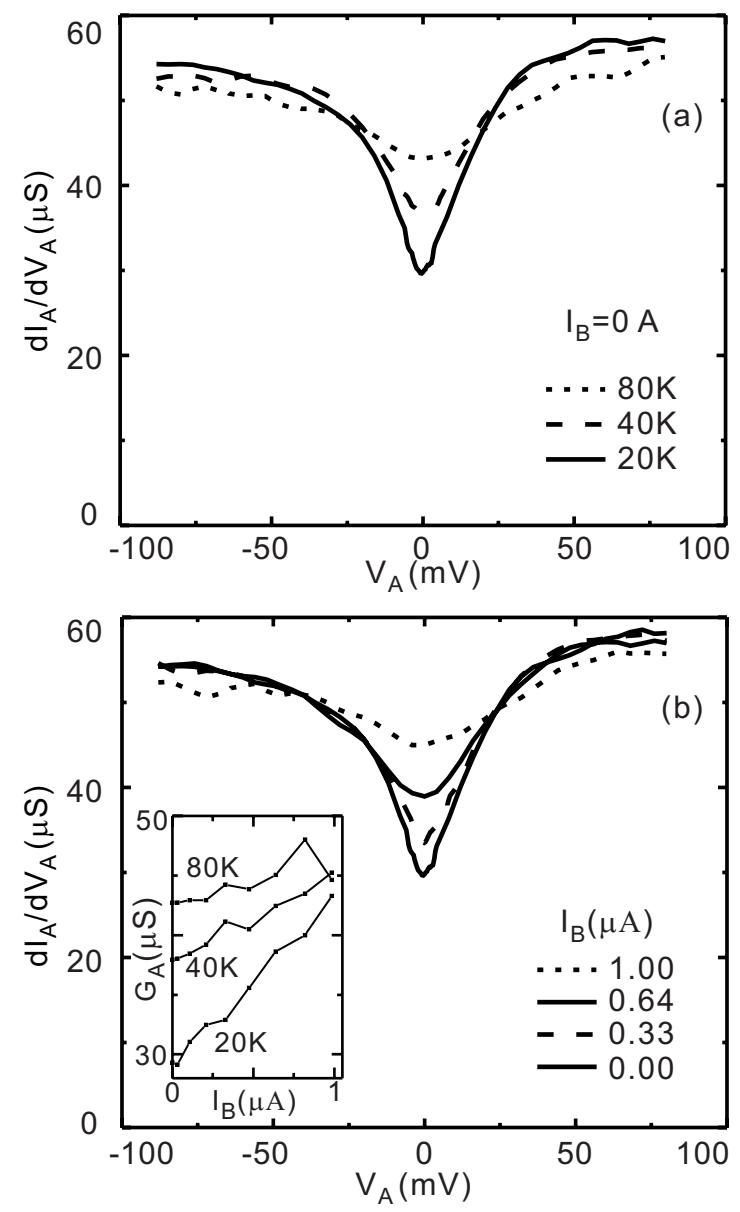

FIG. 3: Differential conductance $d I_{A} / d V_{A}\left(V_{A}\right)$ measured on SWNT $A$ (a) for different $T$ and (b) for different $I_{B}$ through SWNT $B$ at $20 \mathrm{~K}$. The inset in (b) shows $G_{A}$ for $V_{A}=0$ as a function of $I_{B} . I_{B}=1 \mu \mathrm{A}$ corresponds to $V_{B}=0.8 \mathrm{~V}$. The other points are separated in bias by $\Delta V_{B}=0.1 \mathrm{~V}$.

the crossing can be neglected, and hence only tube-tube electrostatic coupling and crossing-induced backscattering (CIB) processes need to be taken into account. The importance of CIB processes due to the tube deformation has been stressed in several previous experimental [19, 24] and theoretical studies [25, 26]. Both are taken as local couplings acting only at the crossing. Adopting the standard bosonization formalism [27], for $g<1 / 5$, the most relevant part of the density operator in tube $\alpha=A, B$ is [4]

$$
\rho_{\alpha}(x) \propto \cos \left[\sqrt{16 \pi g} \varphi_{c+, \alpha}(x)\right],
$$

where $\varphi_{c+, \alpha}$ is the boson field describing charged lowenergy excitations (plasmons) of the SWNT. Choosing spatial coordinates such that $x=0$ corresponds to the crossing point, the Hamiltonian $H=H_{0}+H_{\mathrm{AB}}+H_{\mathrm{CIB}}$ consists of the clean LL part, $H_{0}=\sum_{\alpha} H_{L L, \alpha}$, a local tube-tube coupling $H_{\mathrm{AB}}=\lambda_{0} \rho_{A}(0) \rho_{B}(0)$, and the CIB part $H_{\mathrm{CIB}}=\lambda_{1} \rho_{A}(0)+\lambda_{2} \rho_{B}(0)$. Standard renormalization group (RG) analysis [27] yields the lowest-order flow equations

$$
\begin{aligned}
\frac{d \lambda_{0}}{d \ell} & =(1-8 g) \lambda_{0}+2 \lambda_{1} \lambda_{2}, \\
\frac{d \lambda_{1,2}}{d \ell} & =(1-4 g) \lambda_{1,2},
\end{aligned}
$$

where $\ell$ is the usual flow parameter, $d \ell=-d \ln D$, i.e., one decreases the high-energy bandwidth cutoff $D$ and compensates this decrease by adjusting the couplings. The initial coupling constants $\lambda_{0,1,2}(0)$ could be accessed from microscopic considerations, but here are only assumed to be non-zero. Integration of Eq. (11) yields $\lambda_{1,2}(\ell)=\lambda_{1,2}(0) \exp [(1-4 g) \ell]$ and

$$
\begin{aligned}
\lambda_{0}(\ell) & =\left[\lambda_{0}(0)-2 \lambda_{1}(0) \lambda_{2}(0)\right] e^{(1-8 g) \ell} \\
& +2 \lambda_{1}(0) \lambda_{2}(0) e^{(2-8 g) \ell}
\end{aligned}
$$

Apparently, at low energies (large $\ell$ ), the RG flow is completely dominated by $\lambda_{0}(\ell)$ due to the last term in Eq. (2). Ignoring the couplings $\lambda_{1,2}(\ell)$ at such energy scales is then justified, and one can use the single-channel model with only the $\lambda_{0}$ coupling of Refs. [15, 16], taken at effective interaction parameter $K_{\text {eff }}=4 g-1 / 2$. Taking $g=0.16$, this gives $K_{\text {eff }}=0.14$. For this argument, it is crucial that $g<1 / 5$ and $\lambda_{1,2}(0) \neq 0$, for otherwise $\lambda_{0}$ is irrelevant for all $g>1 / 8$. The CIB processes therefore drive the electrostatic tube-tube coupling $\lambda_{0}$ to be the dominant interaction in this crossed geometry.

The relevancy of the coupling $\lambda_{0}$ now generates a ZBA which disappears when current flows in the second tube, in agreement with experiments. For $K_{\text {eff }}=1 / 4$ (corresponding to $g=0.1875$ ), this can be made explicit by a simple analytical solution of the resulting transport problem [16]. While the exact solution can be obtained for any $K_{\text {eff }}$ as well [28], away from $K_{\text {eff }}=1 / 4$ this solution is less transparent and shows only slight differences. We therefore focus here on $K_{\text {eff }}=1 / 4$, where the current through SWNTs $\alpha=A, B$ is

$$
I_{\alpha}=\frac{4 e^{2}}{h}\left[V_{\alpha}-\left(U_{+} \pm U_{-}\right) / \sqrt{2}\right]
$$

with $U_{ \pm}$obeying the self-consistency relations

$$
e U_{ \pm}=2 k T_{B} \operatorname{Im} \Psi\left(\frac{1}{2}+\frac{k T_{B}+i\left(e V_{ \pm}-e U_{ \pm}\right)}{2 \pi k T}\right)
$$

with the digamma function $\Psi, V_{ \pm}=\left(V_{A} \pm V_{B}\right) / \sqrt{2}$, and an effective coupling strength $T_{B}$, which depends on the system parameters, in particular on the initial couplings $\lambda_{0,1,2}(0)$.

Figures $4(\mathrm{a}-\mathrm{b})$ show modified $d I_{A} / d V_{A}\left(V_{A}\right)$ curves of Fig. 3. Indeed, Fig. 3] shows that the high-bias differential conductance $d I_{A} / d V_{A}$ saturates at $(17.9 \mathrm{k} \Omega)^{-1}$ instead of $4 e^{2} / h$, which is the high-bias conductance predicted by Eqs. (3) and (4). We therefore argue that a resistance $R_{c}=11.4 \mathrm{k} \Omega$ lies in series with the $I_{B}$ dependent contribution of the inter-tube coupling in order to obtain this 

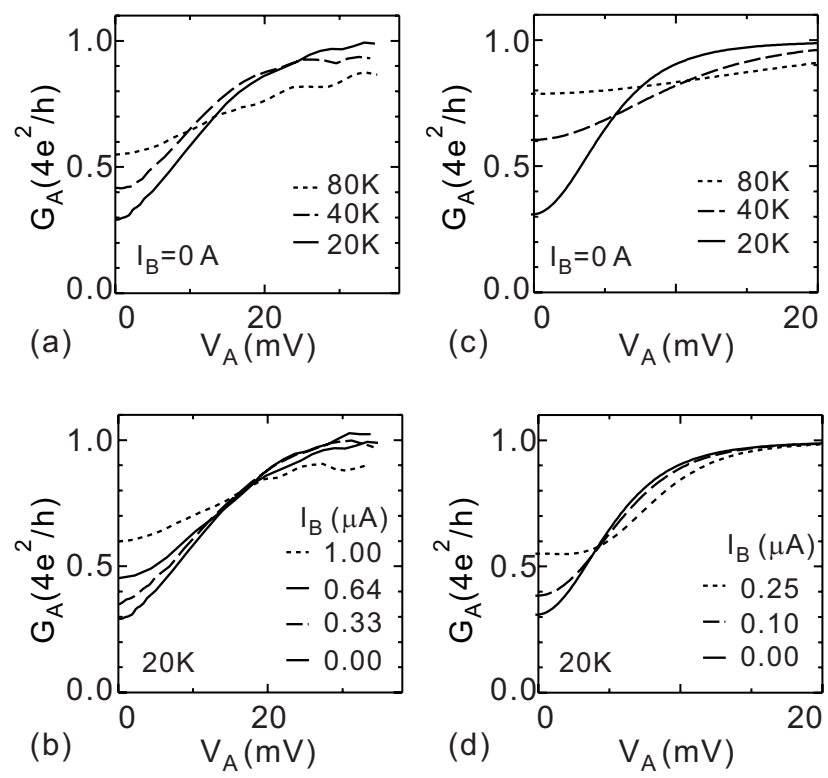

FIG. 4: Differential conductance $d I_{A} / d V_{A}\left(V_{A}\right)$ symmetrized and modified from Fig. 3 for (a) different $T$ and (b) for different $I_{B}$. Theoretical predictions for two interacting SWNTs are shown in (c) and (d). The curves in (d) are obtained for constant biases $V_{B}$. The corresponding currents $I_{B}$, which are calculated with Eqs. (3) and (4) for $V_{A}=0$, are given in the legend.

$d I_{A} / d V_{A}$ saturation. The resistance $R_{c}$, presumably located at the tube-electrode interfaces, is taken constant. This approximation is quite good since the ZBA tends to disappear for large $I_{B}$, leaving only a weak $1 / R_{c}$ con- ductance modulation, see Fig. 3(b). Moreover, the conductance is known to change only slightly with $T$ or $V$ in experiments on individual SWNTs that are well contacted with contact resistance of the order $10 \mathrm{k} \Omega$ [2, 3].

Figs. 4( c,d) show the predicted $d I_{A} / d V_{A}\left(V_{A}\right)$ curves calculated from Eq. (3) with (4). The effective coupling $T_{B}$ is set at $T_{B}=11.6 \mathrm{~K}$ to get agreement with the experimental value for $G_{A}$ at $20 \mathrm{~K}, I_{B}=0$ and $V_{A}=0$. Despite the above-mentioned approximations, the agreement of theory and experiment is quite good. After fixing $T_{B}$, no parameter is tuned to calculate the conductance variation with $V_{A}, T$ and $I_{B}$. We note in passing that Eqs. (34) predict the emergence of minima in $d I_{A} / d V_{A}\left(V_{A}\right)$ at large $I_{B}$, when $I_{B} \gtrsim 1 \mu \mathrm{A}$. These interesting features have not been observed though. One probable cause could be the inelastic scattering on optical phonons, which takes place at such large currents [29]. Scattering processes of this kind are not included in Eqs. (3) and (4).

We have presented experimental data for transport in crossed metallic single-wall carbon nanotubes. The results are in rather good agreement with a theoretical analysis based on the Luttinger liquid model, and cannot be rationalized by several alternative mechanisms. We therefore take this as new evidence for the Luttinger liquid picture of SWNTs beyond tunneling experiments.

We thank B. Plaçais, J.M. Berroir, N. Regnault and C. Delalande for discussions. LPMC is CNRS-UMR8551 associated to Paris 6 and 7 . This research has been supported by ACN programs (NN029), the EU network DIENOW, the DFG-SFB TR 12, and by the Landesstiftung Baden-Württemberg.
[1] A. Bachtold, M.S. Fuhrer, S. Plyasunov, M. Forero, E.H. Anderson, A. Zettl, P. L. McEuen, Phys. Rev. Lett. 84, $6082(2000)$.

[2] J. Kong, E. Yenilmez, T. W. Tombler, W. Kim, H. Dai, R. B. Laughlin, L. Liu, C. S. Jayanthi, S. Y. Wu, Phys. Rev. Lett. 87, 106801 (2001).

[3] W. Liang, M. Bockrath, D. Bozovic, J. H. Hafner, M. Tinkham, H. Park, Nature 411, 665 (2001).

[4] R. Egger and A. O. Gogolin, Phys. Rev. Lett. 79, 5082 (1997).

[5] C. Kane, L. Balents, and M. P. A. Fisher, Phys. Rev. Lett. 79, 5086 (1997).

[6] M. Bockrath, D.H. Cobden, J. Lu, A.G. Rinzler, R.E. Smalley, L. Balents, P. L. McEuen, Nature 397, 598 (1999).

[7] Z. Yao, H.W.Ch. Postma, L. Balents, C. Dekker, Nature 402, 273 (1999).

[8] H.W.C. Postma, M. deJonge, Z. Yao, C. Dekker, Phys. Rev. B 62, R10653 (2000).

[9] H.W.Ch. Postma, T. Teepen, Z. Yao, M. Grifoni, C. Dekker, Science 293, 76 (2001).

[10] A. Bachtold, M. de Jonge, K. Grove-Rasmussen, P. L. McEuen, M. Buitelaar, C. Schönenberger, Phys. Rev. Lett. 87, 166801 (2001).
[11] R. Tarkiainen, M. Ahlskog, J. Penttilä, L. Roschier, P. Hakonen, M. Paalanen, E. Sonin, Phys. Rev. B 64, 195412 (2001).

[12] W. Yi, L. Lu, H. Hu, Z. W. Pan, S. S. Xie, Phys. Rev. Lett. 91, 076801 (2003).

[13] R. Egger and A.O. Gogolin, Phys. Rev. Lett. 87, 066401 (2001).

[14] E.G. Mishchenko, A.V. Andreev, L.I. Glazman Phys. Rev. Lett. 87, 246801 (2001)

[15] A. Komnik and R. Egger, Phys. Rev. Lett. 80, 2881 (1998).

[16] A. Komnik and R. Egger, Eur. Phys. J. B 19, 271 (2000).

[17] M.S. Fuhrer, J. Nygard, L. Shih, M. Forero, Y. G. Yoon, M.S.C. Mazzoni, H.J. Choi, J. Ihm, S.G. Louie, A. Zettl, P.L. McEuen, Science 288, 494 (2000).

[18] J. Kim, K. Kang, J.-O. Lee, K.-H. Yoo, J.-R. Kim, J.W. Park, H.M. So, J.-J. Kim, J. Phys. Soc. Jpn. 70, 1464 (2001).

[19] J.W. Janssen, S.G. Lemay, L.P. Kouwenhoven, C. Dekker, Phys. Rev. B 65, 115423 (2002).

[20] A. Thess, R. Lee, P. Nikolaev, H. Dai, P. Petit, J. Robert, C. Xu, Y.H. Lee, S.G. Kim, A.G. Rinzler, D.T. Colbert, G.E. Scuseria, D. Tomnek, J.E. Fischer, R.E. Smalley, Science 273, 483 (1996). 
[21] S. Berber, Y.K. Kwon, D. Tomanek, Phys. Rev. Lett. 84, 4613 (2000).

[22] Q. Zheng, G. Su, J. Wang, H. Guo, Eur. Phys. J. B 25, 233 (2003).

[23] P. Kim, L. Shi, A. Majumdar, P.L. McEuen, Phys. Rev. Lett. 87, 215502 (2001).

[24] T. Hertel, R.E. Walkup, P. Avouris, Phys. Rev. B 58, 13870 (1998).

[25] A. Rochefort, P. Avouris, F. Lesage, D. R. Salahub, Phys. Rev. B 60, 13824 (1999).
[26] M.B. Nardelli and J. Bernholc, Phys. Rev. B 60, R16338 (1999).

[27] A.O. Gogolin, A.A. Nersesyan, A.M. Tsvelik, Bosonization and strongly correlated systems (Cambridge University Press, 1998).

[28] R. Egger, H. Grabert, A. Koutouza, H. Saleur, F. Siano, Phys. Rev. Lett. 84, 3682 (2000).

[29] Z. Yao, C.L. Kane, C. Dekker, Phys. Rev. Lett. 84, 2941 (2000). 